\title{
Modeling of Scalar Dissipation Rates in Flamelet Models for HCCI Engine Simulation
}

\author{
Saurabh Gupta ${ }^{1}$, SeungHwan Keum ${ }^{2}$, and Hong G. $\mathrm{Im}^{3}$ \\ Department of Mechanical Engineering, University of Michigan, \\ Ann Arbor, MI 48109-2125
}

\begin{abstract}
The flamelet approach is considered a viable framework to the modeling of homogeneous charge compression ignition (HCCI) engines under stratified mixture conditions. However, there are several issues that need further improvement. In particular, accurate representation of the scalar dissipation rate, which is the key parameter to connect the physical mixing space to the reactive space, requires further investigation. This involves a number of aspects: (i) probability density functions, (ii) mean scalar dissipation rates, and (iii) conditional scalar dissipation rates, for mixture fraction $(Z)$ and total enthalpy $(H)$. The present study aims to assess the validity of existing models in HCCI environments both in the RANS and LES contexts, and thereby suggest alternative models to improve on the above three aspects.
\end{abstract}

\section{Nomenclature}

$Z \quad=$ mixture fraction

$H \quad=$ total enthalpy

$\chi_{Z} \quad=\quad Z$ scalar dissipation rate

$\chi_{H} \quad=H$ scalar dissipation rate

$\widetilde{\chi_{Z}}=$ Mean $Z$ scalar dissipation rate

$\widetilde{\chi_{H}}=$ Mean $H$ scalar dissipation rate

$\widetilde{\chi_{Z H}}=$ Mean $Z-H$ cross scalar dissipation rate

$\widetilde{Z^{\prime \prime 2}} \quad=$ Variance of $Z$

$\widetilde{H^{\prime \prime 2}}=$ Variance of $H$

$\tilde{\varepsilon} \quad=$ Mean turbulent kinetic energy dissipation rate

$\tilde{\kappa}=$ Mean turbulent kinetic energy

\section{Introduction}

$\mathrm{H}$ omogeneous charge compression ignition (HCCI) engine technology shows promises as an advanced internal combustion (IC) engines in favor of lower $\mathrm{NO}_{\mathrm{x}}$ and soot emissions and higher efficiencies. This has led to an ongoing interest in HCCI engine research for over than two decades [1,2]. Considering that the mixture field within the HCCI engines is nearly homogeneous, full-cycle engine simulations based on the multi-zone models have been conducted with success [3-6]. More recently, however, mixture stratification by exhaust gas recirculation or residual

\footnotetext{
${ }^{1}$ Graduate Student, Department of Mechanical Engineering, University of Michigan, Ann Arbor, MI 48109, AIAA Student Member.

${ }^{2}$ Present Address: Researcher, Propulsion Systems Research Laboratory, General Motors Technical Center, Warren, MI 48092.

3 Professor, Department of Mechanical Engineering, University of Michigan, Ann Arbor, MI 48109, AIAA Associate Fellow.
} 
gas trap has been extensively considered as a means to achieve combustion phasing control and smoother pressure rise. Therefore, fundamental studies have been conducted to understand combustion characteristics under stratified mixture conditions [7, 8]. Ultimately, these effects must be properly accounted for in engine combustion simulations based on Reynolds-averaged Navier-Stokes (RANS) or large eddy simulation (LES) approaches. As a viable submodel for combustion processes in stratified HCCI conditions, flamelet models have been proposed as an improved alternative to the multizone models $[9,10]$.

Unlike the name may suggest, the flamelet approach [11] should be viewed as a general framework of describing chemical reactions in a reduced-dimensional space in which conserved scalar variables are used as the independent coordinate variables. The multi-dimensional computational fluid dynamics (CFD) simulation only considers transport equations for velocity and nonreactive scalar variables. To accurately describe highly transient events such as auto-ignition encountered in HCCI engines, the representative interactive flamelet (RIF) approach [12] solves the unsteady flamelet equation in the reactive space in conjunction with the time integration of the CFD solver. As for the conserved scalar variables, the mixture fraction, $Z$, is commonly used as the one-dimensional conserved scalar. To account for the nonuniform enthalpy levels due to exhaust gas mixing and heat losses, the total enthalpy variable, $H$, can be added to construct a two-dimensional flamelet equation in the $Z-H$ space [10]. One of the key parameters in the flamelet equation is the scalar dissipation rate, defined as

$$
\chi_{Z}=2 \alpha|\nabla Z|^{2}
$$

where $\alpha$ is the diffusivity. For the two-dimensional flamelet approach, additional scalar dissipation rate for $H$ needs to be defined as

$$
\chi_{H}=2 \alpha|\nabla H|^{2}
$$

The scalar dissipation rate is the key parameter that connects the physical transport space and the reactive space. At each time step of the numerical solution, at first the equations in the physical space are solved to obtain the mean scalar dissipation rate at stoichiometric conditions. This is then used to calculate the conditional scalar dissipation rate through an analytical model $[13,14]$. Conditional scalar dissipation rate is further used as an input to the flamelet equations (in the transformed space). The output parameters from the transformed space (species mass fractions as a function of $Z$ and $H$ ) are then fed into the physical space to complete one time step cycle.

Although flamelet models have been successfully used in modeling HCCI combustion under stratified conditions, there still remain open issues. In particular, there still has not been a thorough study to investigate modeling of the scalar dissipation rate. Modeling scalar dissipation rate involves a number of aspects, the most important being the probability density functions, mean scalar dissipation rates, and conditional scalar dissipation rates for $Z$ and $H$.

Therefore, the objectives of the present study are to validate various existing models, and to propose improved alternatives for:
a. Probability density functions of $Z$ and $H$
b. Mean $Z$ and $H$ scalar dissipation rates
c. Conditional $Z$ and $H$ scalar dissipation rates

As an a priori test, two-dimensional direct numerical simulation (DNS) of auto-ignition of a turbulent $\mathrm{H}_{2}$-air mixture with detailed chemistry has been used for validation [15]. DNS simulations considered four parametric cases in terms of mixture inhomogeneities imposed as the initial condition:

a. Temperature inhomogeneities only (CaseA)

b. Uncorrelated temperature and compositional inhomogeneities (CaseB)

c. Negatively-correlated temperature and compositional inhomogeneities (CaseC)

These cases represent vastly different combustion modes (homogeneous ignition, premixed flame propagation and sequential ignition). Therefore, the DNS data serve as a good reference to test the models on a multitude of different engine operation scenarios.

The first section of this paper deals with the validation of the existing models for pdfs of $Z$ and $H$. In the second section, we validate the existing model and propose a new model for mean $Z$ and $H$ scalar dissipation rates. Next, we investigate the cross scalar dissipation rates, and propose model improvements for them. Subsequently, the performance of the existing models in the context of LES will be examined. The key findings from this study are summarized in the conclusions section. 


\section{Probability density functions for $Z$ and $H$}

In this study, the normalized values of mixture fraction and enthalpy variables are used based on their minimum and maximum values at each time step. Normalization is done in the flamelet approach in order to simplify numerical computations in the flamelet space [18]. The probability density function (pdf) is calculated using the DNS data, as reference to validate the pdf models.

Figures 1a and 1c show the pdf of $Z$ (calculated from DNS data) for cases $\mathrm{B}$ and $\mathrm{C}$, respectively. Pdfs of $H$ follow similar trend and so have not been shown here. Cases B and $\mathrm{C}$ have been chosen as they represent different combustion modes (Case B favors ignition front propagation, whereas case $\mathrm{C}$ favors homogeneous volumetric ignition [15]. For case B (Figure 1a), pdf starts off symmetrically (at $\mathrm{t}=0 \mathrm{~ms}$ ) with its maximum value at $Z=0.5$. During the course of combustion, the peak of pdf shifts left towards lower $Z$ values, reaches a minimum and then again shifts right towards the end of combustion. However, for case C (Figure 1c), no such shift in the peak is observed. Hence, one can expect that this peak shifting is related to the mode of combustion.

In case B, ignition fronts form at around $1.5 \mathrm{~ms}$ (Figure 2a), which causes a sudden gradient in mixture composition, with the ignition front separating the unburned and the burning region. As can be seen from Figure 2, there are sharp gradients in $Z$ as well, and although not shown here, $\boldsymbol{H}$ also undergoes a similar type of behavior. At this point, it is very important to understand the reason behind the appearance of these gradients, because they cause a sudden rise in the local scalar dissipation rate, which consequently poses a lot of modeling challenges. Dealing with such modeling issues is the central theme of this study.

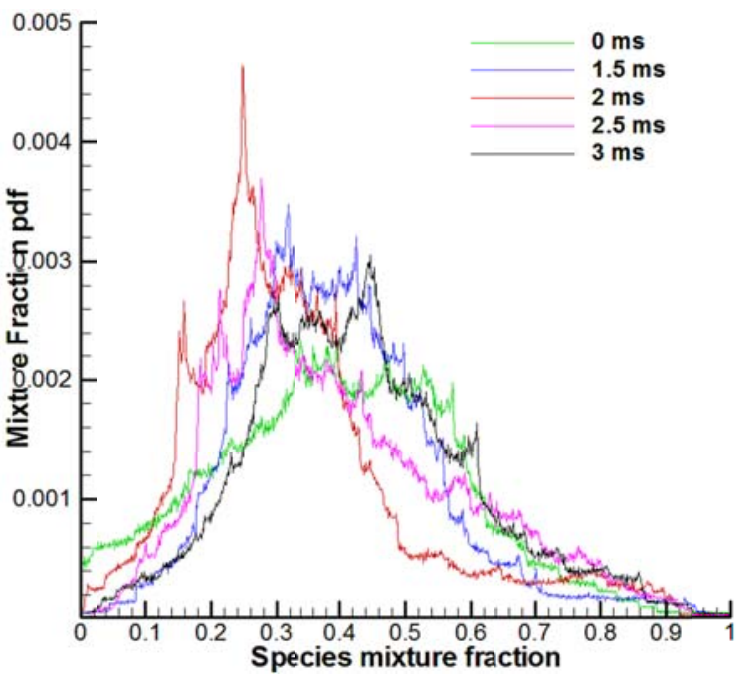

Figure 1a. Pdf of $Z$ calculated from DNS data (CaseB)

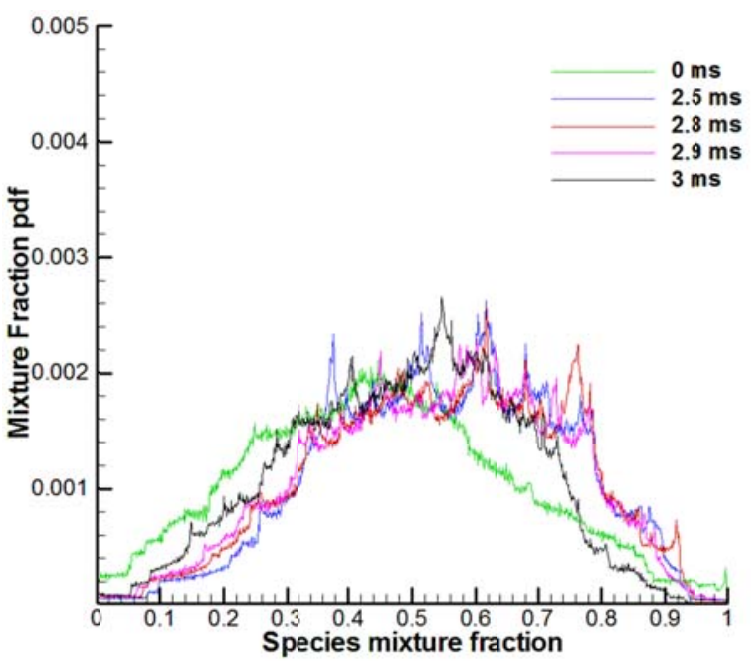

Figure 2c. Pdf of $Z$ calculated from DNS data (CaseC)

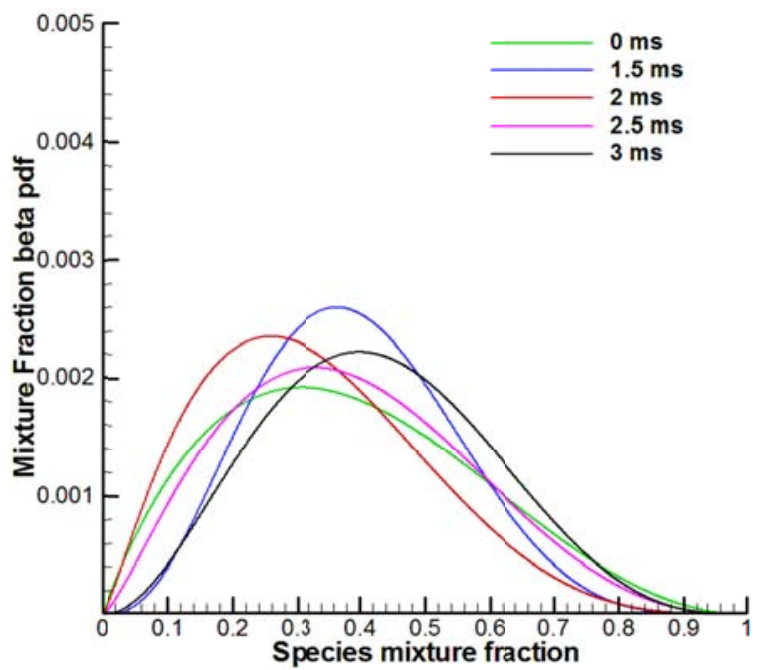

Figure 1b. Beta pdf of $Z$ (Case B)

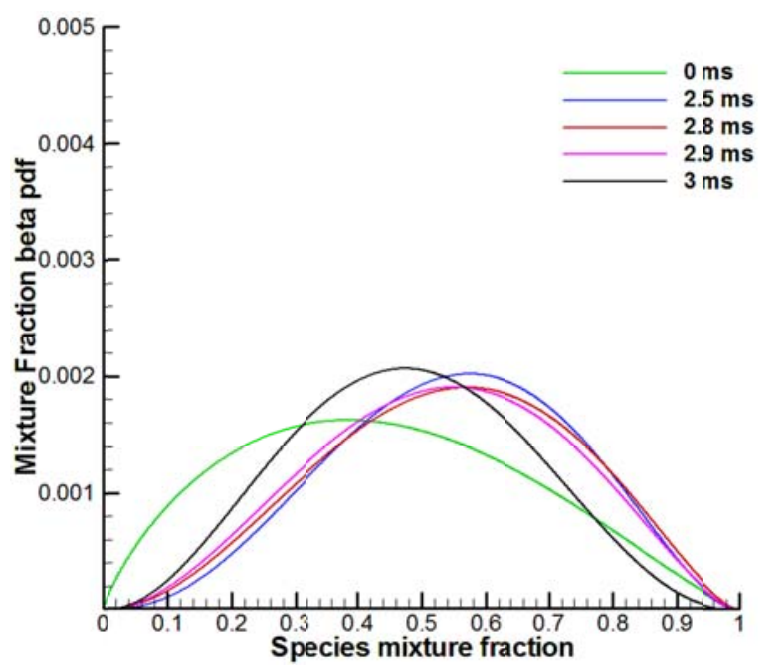

Figure 1d. Beta pdf of $Z$ (Case $C$ ) 
The appearance of sharp gradients might at a first sight seem contradictory to the definition of a conserved scalar, as by definition, a conserved scalar cannot be generated by combustion. In fact, the volume averaged $\mathbf{Z}$ does not undergo any change at all during the course of combustion. Volume averaged $\boldsymbol{H}$, however, does increase a little bit during heat release, because of the $\mathrm{dp} / \mathrm{dt}$ term in its transport equation. As described next, the main reason has to do with the physical and chemical properties of hydrogen $\left(\mathrm{H}_{2}\right)$.

Hydrogen has a Lewis number much less than unity, and thus can diffuse much faster as compared to other species. As soon as the ignition fronts form, hydrogen from the unburned mixture diffuses to the burned mixture, hence increasing the local $Z$ value for the burning region, and decreasing it for the unburned region. Since hydrogen also has a high value of chemical enthalpy, the total enthalpy of burning region increases as soon as hydrogen diffuses in. Similarly, because of the loss of hydrogen, the total enthalpy of the unburned region reduces.

The differential diffusion of hydrogen is also found to be responsible for the shift in pdf peaks. Figure 1a shows that the peaks shift leftwards up until $2 \mathrm{~ms}$. From $\mathrm{t}=1.5 \mathrm{~ms}$ onwards, since the ignition front has formed, the $Z \& H$ values within the unburned region decrease, as explained before. Because most part of the mixture is still unburned, there is a high probability within the domain of finding low $Z$ and low $H$ values. Hence, the peak of the pdf shifts leftwards. But after $2 \mathrm{~ms}$, since the amount of unburned mixture is continuously decreasing, there is now a higher probability of finding moderately higher $Z \& H$ values. This is the reason why after $2 \mathrm{~ms}$, the peak shifts rightwards until it reaches the symmetry position towards the end of the combustion event.

For case $\mathrm{C}$ (Figure 3), which favors more of volumetric ignition, there are no sharp local gradients in $Z$ ( $H$ has similar trends, hence not shown here), and hence no such shifts in pdf peak are observed. (Figure 1c)

Let us now look into modeling of the pdfs. The most commonly used model is the beta pdf model [19]. Figures 1b \& 1d show the beta pdf for case B and case C, respectively. It can be seen that there is a good agreement between the DNS and the beta pdf model, especially for case C. For case B, however, there is a little underprediction by the model at $2 \mathrm{~ms}$ and $2.5 \mathrm{~ms}$. This is mainly because of the occurrence of small length scale ignition fronts, which create large local gradients in $Z$ and $H$. Since beta pdf scales as the volumetric mean and variance of $Z$ (and $\mathrm{H}$ ), it is difficult to obtain an accurate scaling to account for these local gradients. Overall, the beta pdfs provide reasonably accurate results and can safely be used for modeling pdfs of $Z$ an $H$.

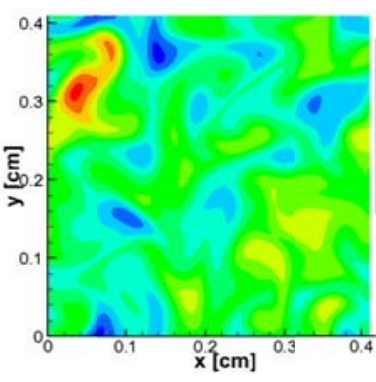

Figure 2a. $1 \mathrm{~ms}$

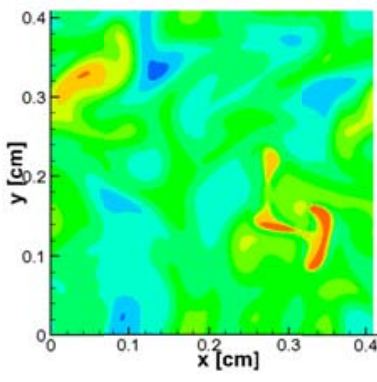

Figure 2b. $1.5 \mathrm{~ms}$

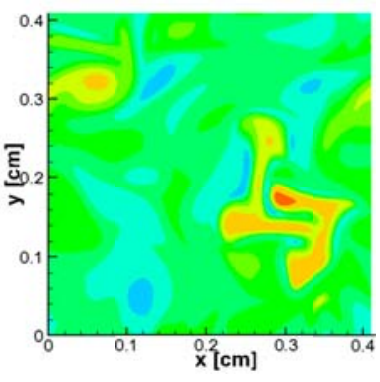

Figure 2c. $2 \mathrm{~ms}$

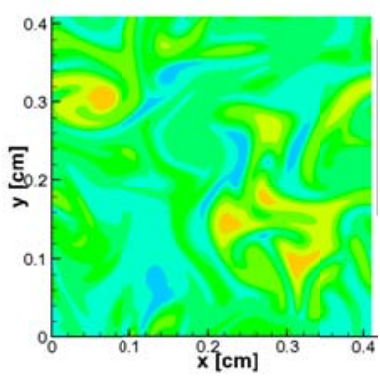

Figure 2d. 2.5ms

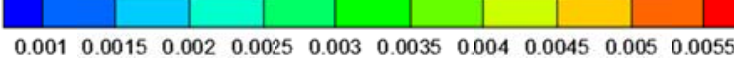

Figure 2. Mixture fraction (non-normalized) at various time instants (Case B)

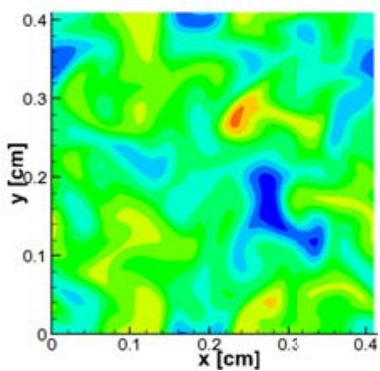

Figure 3a. 1ms

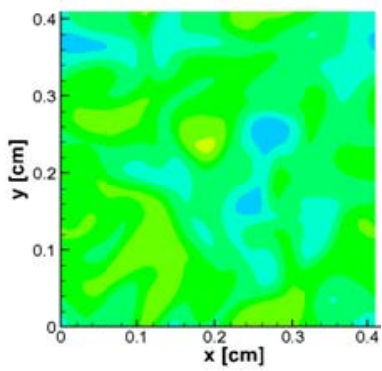

Figure 3b. $2 \mathrm{~ms}$

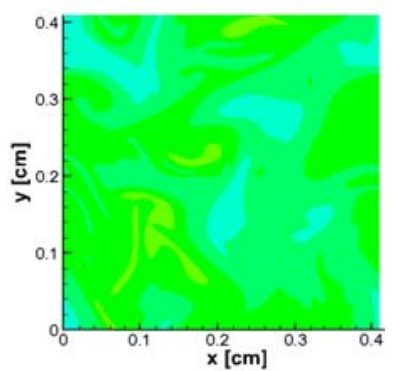

Figure 3c. 3ms

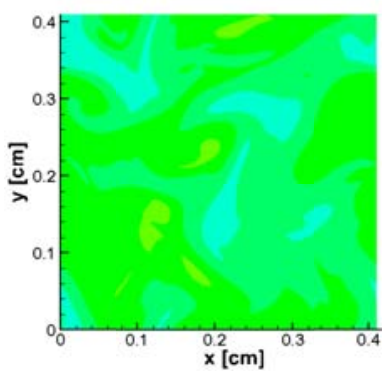

Figure 3d. 3.2ms

$\begin{array}{lllllllllll}0.001 & 0.0015 & 0.002 & 0.0025 & 0.003 & 0.0035 & 0.004 & 0.0045 & 0.005 & 0.0055\end{array}$

Figure 3. Mixture fraction (non-normalized) at various time instants (Case C)

4

American Institute of Aeronautics and Astronautics 


\section{Modeling of mean scalar dissipation rates}

Mean scalar dissipation rates appear as an unclosed term in the transport equations of variances of $Z$ and $H$. They appear not only in the context of flamelet modeling, but many others modeling approaches as well [18]. The classical model which is the most widely used currently is the constant mixing to turbulent timescale model. [18, 19]

$$
\begin{aligned}
& \widetilde{\chi_{Z}}=C_{Z} \frac{\tilde{\varepsilon}}{\tilde{\kappa}} \widetilde{Z^{\prime \prime 2}} \\
& \widetilde{\chi_{H}}=C_{H} \frac{\tilde{\varepsilon}}{\tilde{\kappa}} \widetilde{H^{\prime \prime 2}}
\end{aligned}
$$

where is the constant of proportionality $\left(C_{Z}\right.$ and $\left.C_{H}\right)$ is the ratio of turbulent and mixing timescales, which is generally assumed to be 2.0. This model is first tested against the DNS data.

Figure 4 shows the value of the constant for the three cases. It is observed that $C_{Z}$ and $C_{H}$ are almost constant in the non-reacting regime for all the cases (except for the initial rise which is due to turbulent straining of the mixing field, as an artifact of the initial turbulence seed), although the value of the constant is different for each case, and also different for $Z$ and $H$. We also see that $C_{H}$ is always greater than $C_{Z}$ by a factor of almost 2-3 (except for case A without initial $Z$ fluctuations).

In the reacting regime, $C_{Z}$ and $C_{H}$ are no longer a constant and have a non monotonic behavior for all the three cases, more so for cases A and B which favor ignition front formation. Such a huge deviation from the constant value may lead to an erroneous prediction of the mean scalar dissipation rate in the full cycle RANS simulations, leading to an inaccurate prediction of the ignition delay for such cases. Hence, the classical model doesn't perform quite well during combustion,

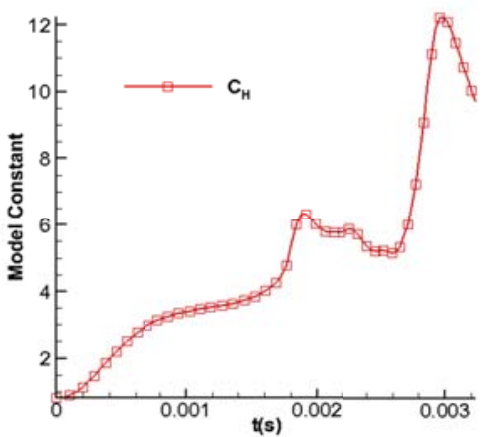

Figure 4a. case A

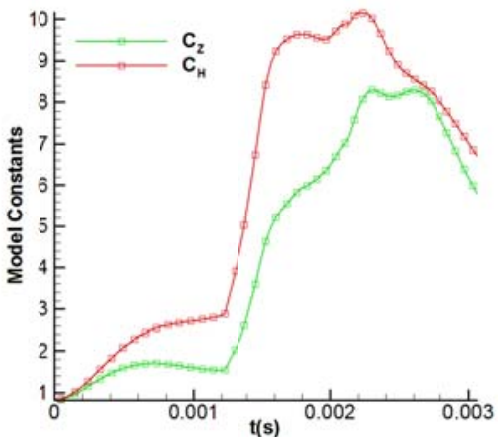

Figure 4b. case $B$

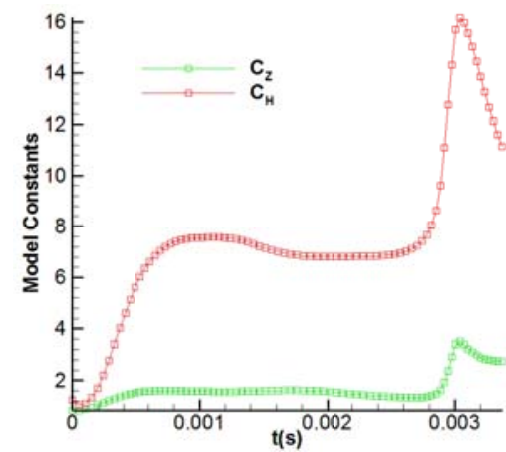

Figure 4c. case $\mathrm{C}$

Figure 4. Model constants $\left(C_{Z}\right.$ and $\left.C_{H}\right)$ for the three cases

Before exploring alternative modeling strategies, we first look into the behavior of the mixing $\left(\tau_{\mathrm{Z}}, \tau_{\mathrm{H}}\right)$ and turbulent $\left(\tau_{\text {turb }}\right)$ timescales for all the three cases (Figure 5) in order to better understand the reasons of poor performance of the classical model. Also plotted is the logarithm of the inverse of Integrated Heat Release rate as a marker of the ignition event.

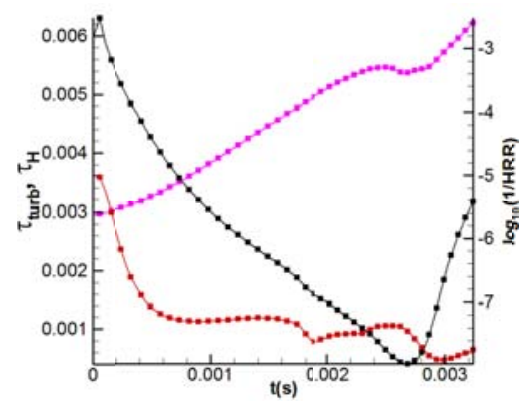

Figure 5a. case $A$

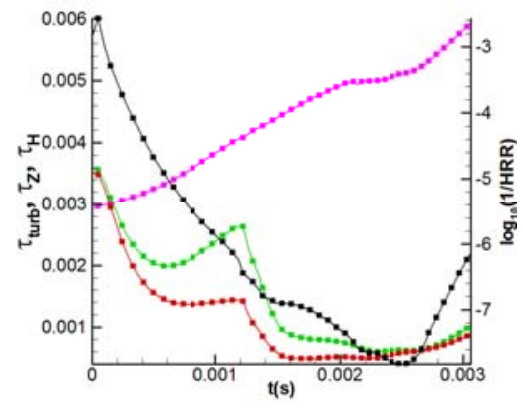

Figure 5b. case B

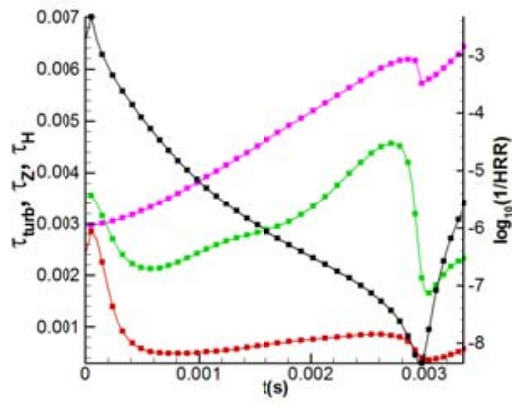

Figure 5c. case $\mathbf{C}$

Figure 5. Timescales for the three cases. Purple: Turbulence time scale, Green: Z Mixing time scale, Red: H Mixing time scale, Black: $\log _{10}(1 /$ Heat Release Rate) 
The following trend is observed in all the cases. The initial turbulence seed causes turbulent straining of the mixing field, resulting in the decrease of mixing timescales. After sometime, when the effect of initial turbulence straining has diminished, the mixture starts to become less inhomogeneous, thereby increasing the mixing timescales. The mixing timescales increase until a point when the ignition fronts start to form inside the domain (notable especially for case A (Figure 5a) and case B (Figure 5b) at $2 \mathrm{~ms}$ and $1 \mathrm{~ms}$, respectively). As explained earlier in the previous section, as soon as the ignition fronts form, there is a large increase in the gradients of both $Z$ and $H$, and hence the scalar dissipation rates increase, causing the mixing timescales to decrease suddenly. From this point onwards until the global volumetric ignition is reached, the mixing timescales are small, and finally increase marginally towards the end when the spatial gradients within the domain decrease towards the end of combustion. For case $\mathrm{C}$, however, which favor volumetric ignition, there is no sudden decrease of mixing timescales.

Therefore, it is clear that the increase in gradients of $Z$ and $H$ for cases $\mathrm{A}$ and $\mathrm{B}$ is the cause for poor performance of the existing models. As explained earlier in section II, this increase in gradients is because of the diffusion of Hydrogen from unburned to burning mixture, faster than the diffusion of other radicals from the burning to unburned region. In order to verify this, we discard the differential diffusion effect by taking the Lewis numbers of all species as 1.0, and run the simulations for cases $\mathrm{A}, \mathrm{B}$ and $\mathrm{C}$ all over again.

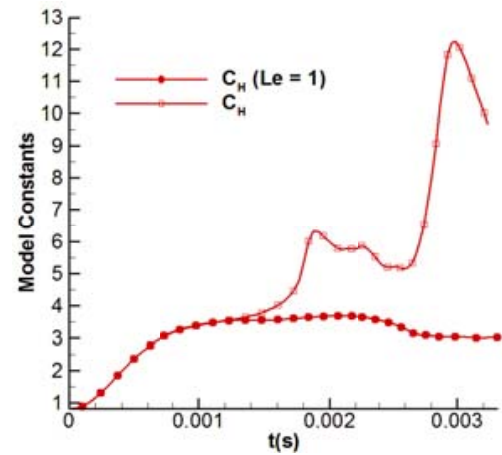

Figure 6a. case A

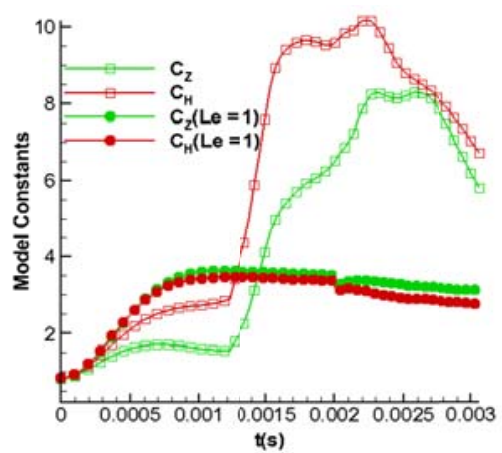

Figure 6b. case B

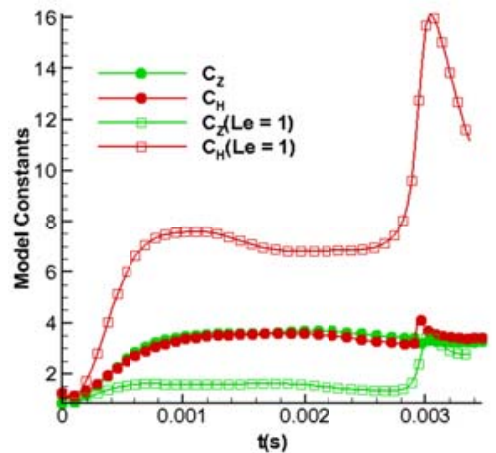

Figure 6c. case $\mathbf{C}$

Figure 6. Model constants $\left(C_{Z}\right.$ and $\left.C_{H}\right)$ for the three cases with (squares) and without (circles) differential diffusion

From Figure 6, one can see that by discarding differential diffusion, $\boldsymbol{C}_{Z}$ and $\boldsymbol{C}_{\boldsymbol{H}}$ more or less attain the value of 3.0 throughout the ignition event. This implies that the flamelet model should also account for the differential diffusion by taking into account non-unity Lewis numbers for all species [22]. The results also suggest that even with the unity Lewis number assumption for all species, the model constants should be taken as 3.0 instead of 2.0.

\section{Modeling of conditional scalar dissipation rates}

In the context of flamelet modeling, conditional scalar dissipation rates are play a crucial role. There are two parts to the conditional scalar dissipation rate. The first one is the scaling factor and the seond one is the assumed functional form of mixture, $\mathrm{f}(Z)[18]$.

Generally $\mathrm{f}(Z)$ is modeled either as a $1 \mathrm{D}$ infinite mixing layer, or as a counterflow flame [18]. Here we present the results for the first (1D infinite mixing layer formulation) model. The results of the second model are almost the same. Figure 7 shows the conditional scalar dissipation rate profile for various time instants for case B, corresponding to both the DNS (Figure 7a) and the model (Figure 7b). It can be seen that during the ignition front propagation period $(1.5 \mathrm{~ms}$ to $2.5 \mathrm{~ms})$, the DNS profiles have considerably higher peak values of the scalar dissipation rate as compared to the model profiles. The increase in peak values is due to the increase in gradients of $Z$ and $H$ during ignition front propagation; which as explained earlier is because of the differential diffusion. If we discard the differential diffusion (Figure 8), we do see that the peak values do not shoot up during ignition front propagation.

It is important to note that the scalar dissipation rate profiles get skewed towards higher mixture fraction values (Figure 7), whereas the same cases without differential diffusion (Figure 8) clearly show no such skewness. This implies that in addition to contributing for the increase in peak values, differential diffusion is also responsible for the skewness of scalar dissipation rate profiles. 
Also, when the differential diffusion is not accounted for, the shape of the scalar dissipation rate profile from DNS (Figure 8a) is a little different from that of the model (Figure 8b). The DNS profile is twin-peaked at the lower and higher values of mixture fraction; whereas the model profile peaks at the center. This implies that we need to have a different profile for the conditional scalar dissipation rate under HCCI conditions. Finding such an appropriate profile is the subject of our future research.

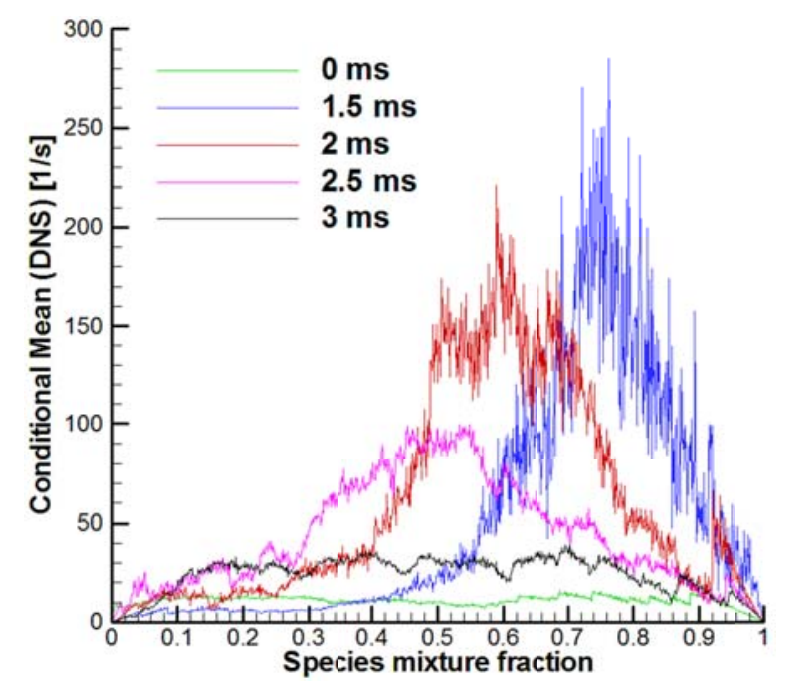

Figure 7a

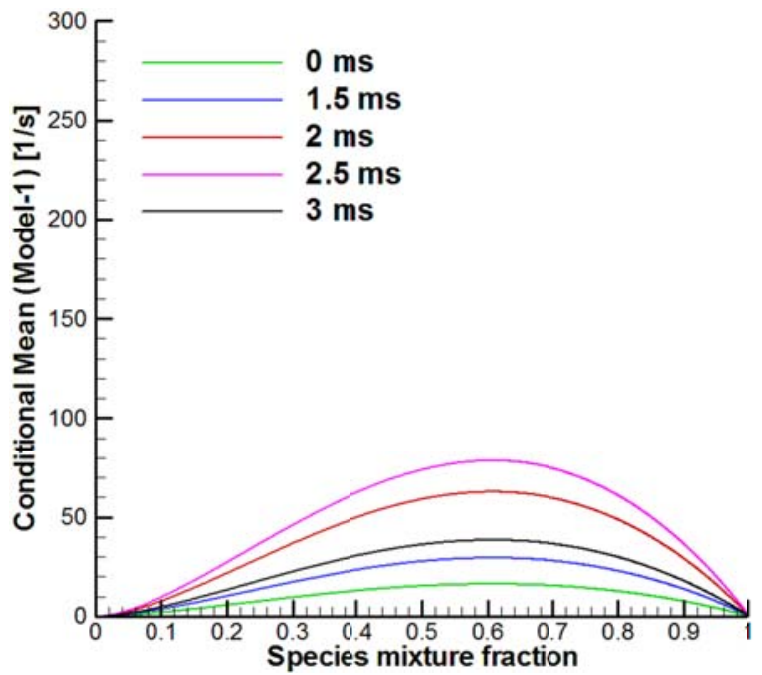

Figure 7b

Figure 7. Conditional $\mathbf{Z}$ scalar dissipation rates calculated from DNS (Figure 7a), and the 1D-infinite mixing layer model (Figure 7 b) for case $B$
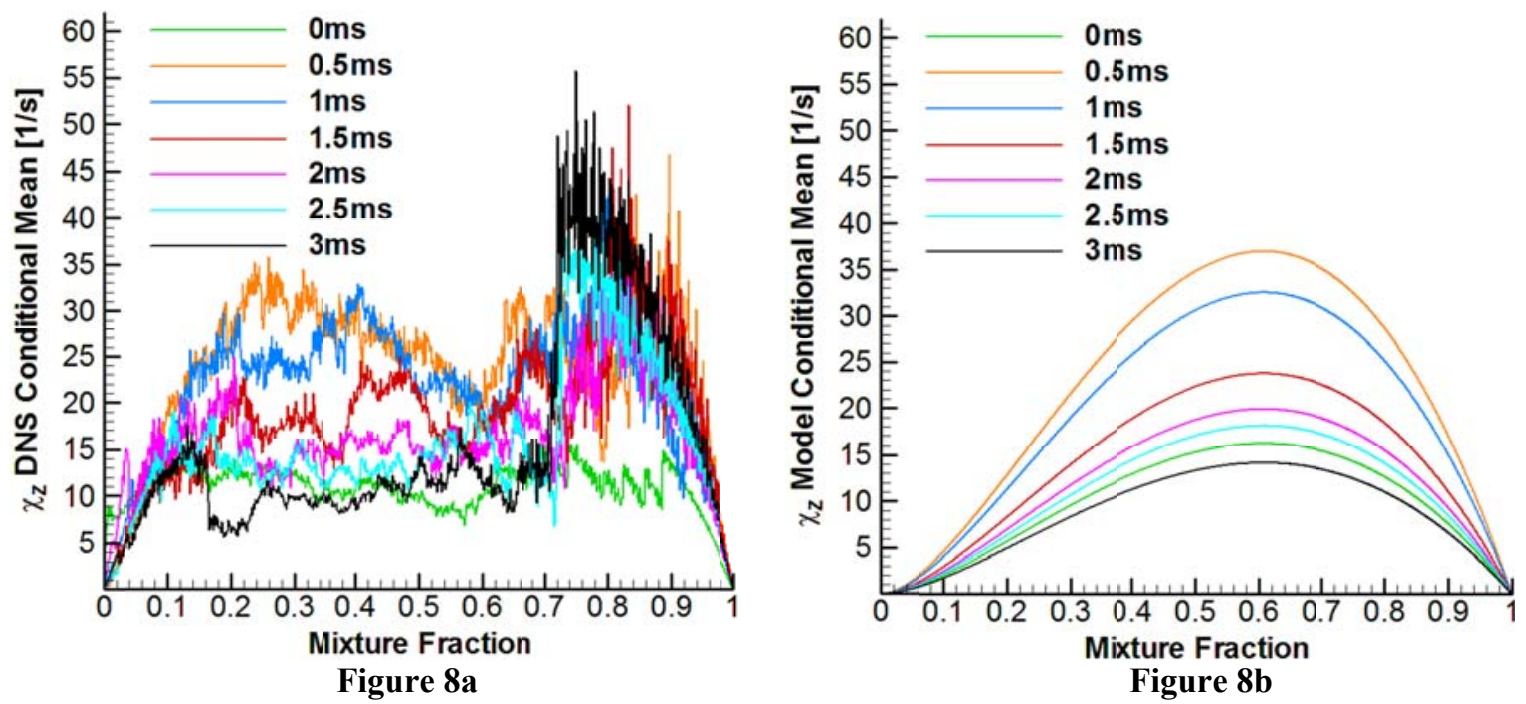

Figure 8. Conditional $\mathbf{Z}$ scalar dissipation rates calculated from DNS (Figure 8a), and the 1D-infinite mixing layer model (Figure 8b) for case B without differential diffusion(L $\boldsymbol{e}_{\boldsymbol{i}}=1$ )

\section{Modeling of scalar dissipation rates for LES}

For all the results discussed so far, the filter size (or averaging size) has been the entire volume. However, if smaller filter sizes are considered, the results could be relevant to LES. This is because in LES, large eddies are resolved, and only the smaller ones need to be modeled. Therefore, the analysis is extended to LES by decreasing the filter size. Generally, the LES filter size is such that it is able to capture the large energy containing eddies, but it is not large enough to properly capture the scalar dissipation rate [21]. Figure 9 shows the typical profile of scalar dissipation rate, extracted from case B at $2 \mathrm{~ms}$. Its thickness is around $0.0058 \mathrm{~cm}$. Hence, if the filter size is larger than this value, the present results should be applicable to LES. 
In this analysis, the filter size was taken to be 30 times that of the DNS grid size at $0.0128 \mathrm{~cm}$ (more than twice the size of the scalar dissipation rate thickness). Figure 10a and 10b show the correlation of the $\mathrm{Z}$ and $\mathrm{H}$ variance with the corresponding mean scalar dissipation rates. The results suggest that mean $\mathrm{Z}$ and $\mathrm{H}$ scalar dissipation rates could be easily modeled as:

$$
\begin{aligned}
& \widetilde{\chi_{Z}}=C \widetilde{Z^{\prime \prime 2}} \\
& \widetilde{\chi_{H}}=C \widetilde{H^{\prime \prime 2}}
\end{aligned}
$$

with $C=10^{4.2}(1 / \mathrm{s})$. It is also seen that turbulence is not at all correlated with the mean scalar dissipation rate (Figure 11). This suggests that the energy containing turbulence length scales are much larger than the filter size, and so are not able to affect mixing in any way. This simple model implies that the mixing timescales for $Z$ and $H$ are constant and moreover have the same value. This might not be a surprise, as we see that in case A (with filter size as the entire volume), the $Z$ mixing timescale remains almost constant throughout the ignition event (Figure 12). Although this case did not have any initial $Z$ fluctuations imposed, having a constant mixing timescale means that without the presence of initial fluctuations, mixing is neither affected by turbulence (during the period right after turbulent straining), nor by the volumetric ignition (towards the end of ignition). Similar explanation holds true when the filter size is comparable to LES.

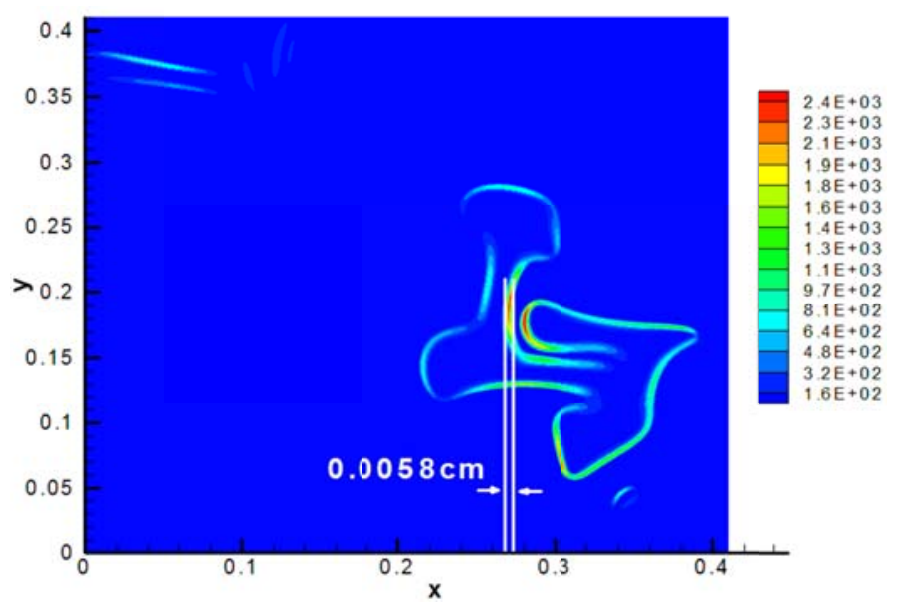

Figure 9. Characteristic thickness of $\mathbf{H}$ scalar dissipation rate (2ms, case B)

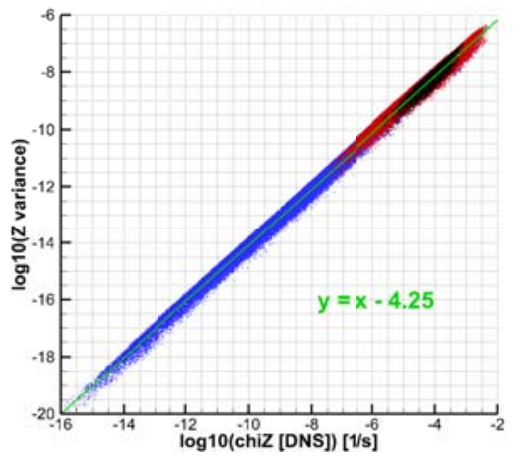

Figure 10a

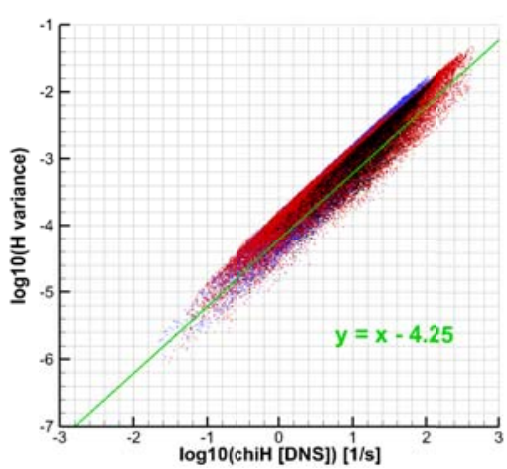

Figure 10b

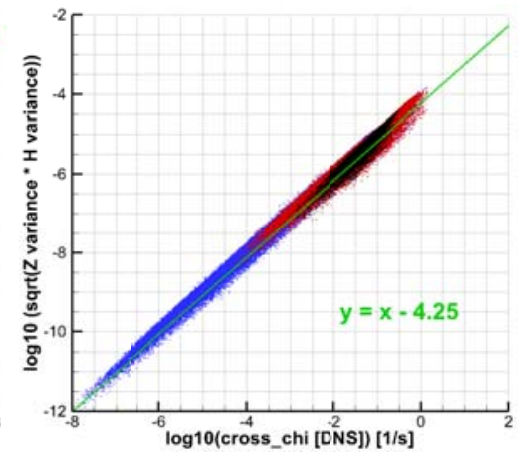

Figure 10c

Figure 10. Mean scalar dissipation rates (filter size $=30 * \Delta_{\text {DNS }}$, case $\mathbf{A}$ ), for $Z$ (Figure 10a), $\boldsymbol{H}$ (Figure 10b), Z-H cross (Figure 10c) 


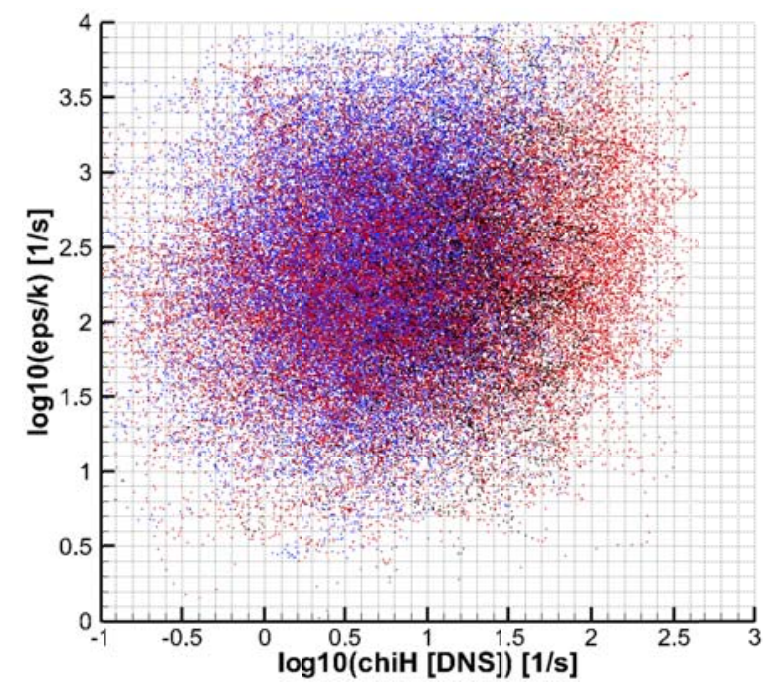

Figure 11. Correlation of inverse eddy turnover time with $\mathrm{Z}$ mean scalar dissipation rate (filter size $=30 * \Delta_{\mathrm{DNS}}$, case $\mathrm{A}$ )

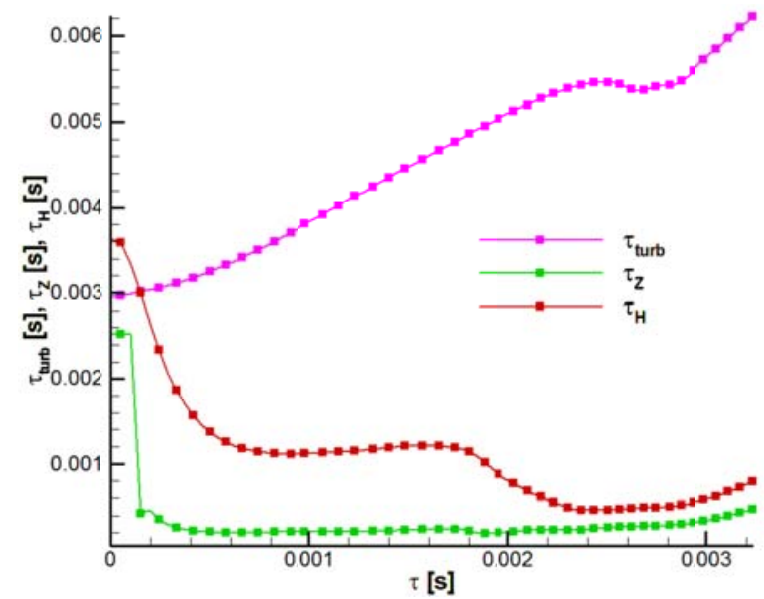

Figure 12. Mixing and Turbulent timescales (Case A). $\tau_{Z}$ is almost constant throughout the entire ignition event.

Based on the above models for mean $Z$ and $H$ scalar dissipation rates, a very simple model can be created for the mean cross scalar dissipation rate, which is an unclosed term in the $2 \mathrm{D} Z-H$ flamelet formulation.

$$
\widetilde{\chi_{Z H}}=C \sqrt{\widetilde{Z^{\prime \prime 2}} \widetilde{H^{\prime \prime 2}}}
$$

From Figure 10c, we see that this model performs extremely well with the same value of the constant $C$ as earlier.

\section{Conclusions}

This study provides fundamental insights into development of mixing models for RANS and LES modeling of stratified HCCI combustion using the flamelet approach. The reference DNS data pertains to a 2D turbulent constant volume problem with thermal and compositional inhomogeneities for hydrogen-air system.

In the RANS context, we first looked at the existing models for pdfs of $Z$ and $H$. It was found that beta pdfs of $Z$ and $H$ give sufficiently accurate results, and thus can be safely used in the scalar dissipation rate modeling. Next, we looked at the existing model for the mean scalar dissipation rates, which is based on mixing timescale being proportional to the turbulent timescale, the constant of proportionality being $C=\tau_{\text {turb }} / \tau_{\text {mix }}$. We plotted $C$ for various test cases representing different ignition modes, and observed that $C$ attains a constant value for the non reacting 
regime, but as soon as the ignition fronts form, it becomes non-monotonic. The reason was identified as the increase in local scalar dissipation rate due to differential diffusion of hydrogen from the unburned to burning region. To verify that differential diffusion is indeed the cause, we ran the same test cases with unity Lewis numbers for all species, and found the constant of proportionality to be 3.0 for both $Z$ and $H$ throughout the entire ignition event. This implies that differential diffusion plays a very crucial role in mixing, and it shold be properly accounted for in the the flamelet equations. The results also suggest that even with the unity Lewis number assumption for all species, $C$ should be taken as 3.0 instead of 2.0 under HCCI conditions.

The conditional scalar dissipation model development has two-fold challenges: a) To properly account for the scaling factor, and $\mathrm{b}$ ) To account for the shape of the assumed function $\mathrm{f}(Z)$. By discarding the differential diffusion, the scaling factor was properly accounted for by the 1D-infinite mixing layer model. Differential diffusion was also found to be responsible for the skewness of scalar dissipation rates towards higher $Z$ values. It was identified that the shape of the DNS profile is twin-peaked, whereas the model profile has a single peak at the center. To propose a suitable profile to combat this inconsistency is our future research work.

For filter size corresponding to LES, it was revealed that turbulence is completely uncorrelated with mixing, and that the mean scalar dissipation rates can be safely modeled as being proportional to the corresponding scalar variances, the constant of proportionality turning out to be the same for $Z$ and $H$. Based on this, a new model for cross scalar dissipation rate was proposed and it gave excellent correlations.

\section{Acknowledgments}

This work was sponsored by the Department of Energy under the University Consortium on Low Temperature Combustion for High Efficiency, Ultra-Low Emission Engines, directed by the University of Michigan under agreement DE-FC26-06NT42629. The computational resources for the 2-D DNS simulations were supported in part by the National Science Foundation through TeraGrid provided by Pittsburgh Supercomputing Center. The authors would like to thank Dr. Gaurav Bansal for providing the simulation data.

\section{References}

${ }^{1}$ P.M. Najt, D.E. Foster, SAE Paper 830264, 1983

${ }^{2}$ F. Zhao, T.W. Asmus, D.N. Assanis, J.E. Dec, J.A. Eng, P.M. Najt (Eds.), Society of Automotive Engineers, Warrendale, PA, 2003

${ }^{3}$ T. Noda, D.E. Foster, SAE Paper 2001-01-0250, 2001

${ }^{4}$ S. B. Fiveland, D. N. Assanis, SAE Paper 2001-01-1028, 2001

${ }^{5}$ W.L. Easely, A. Agarwal, G.A. Lavoie, SAE Paper 2001-01-1029, 2000

${ }^{6}$ N. P. Komninos, D.T. Hountalas, D.A. Kouremenos, SAE Paper 2004-01-0562, 2004

${ }^{7}$ J. H. Chen, E. R. Hawkes, R. Sankaran, S. D. Mason, H. G. Im, Combust. Flame 145 (2006) 128-144

${ }^{8}$ E. R. Hawkes, R. Sankaran, P. P. Pébay, J. H. Chen, Combust. Flame 145 (2006) 145-159

${ }^{9}$ D. J. Cook, H. Pitsch, J. H. Chen, E. R. Hawkes, Proc. Combust. Inst. 31 (2007) 2903-2911

${ }^{10}$ D. J. Cook, H. Pitsch, G. Nentwig, SAE Paper 2008-01-1666, 2008

${ }^{11}$ N. Peters, Prog. Energy Combust. Sci., 10 (1984) 319-339

${ }^{12}$ H. Pitsch, Y. P. Wan, and N. Peters, SAE Paper 952357, 1995

${ }^{13}$ N. Peters, Cambridge University Press, Cambridge; New York 2000

${ }^{14}$ H. Pitsch, H. Barths, N. Peters, SAE Paper 962057, (1996)

${ }^{15}$ G. Bansal, H.G. Im, AIAA 2009-1559, (2009)

${ }^{16}$ R. W. Bilger, S. H. Starner, Combust. Flame 80 (1990) 135-149

${ }^{17}$ A. Kobiera, H. G. Im, V. Hamosfakidis, Proceedings of the International COST Workshop, 2009

${ }^{18}$ D. J. Cook, PhD Thesis, Stanford University, (2007)

${ }^{19}$ S. H. Keum, PhD Thesis, University of Michigan, (2009)

${ }^{20}$ Mastorakos, E., Baritaud, T. A., and Poinsot, T. J., Combust. Flame 109:198-223 (1997).

${ }^{21}$ Y. Zhang, J. Ghandhi, B. Petersen, C. J. Rutland, SAE Paper 2010-01-0625, (2010)

${ }^{22}$ H. Pitsch and N. Peters, Combust. Flame 114 (1998) 26-40 